\title{
UNA NOTA SOBRE LOS SETENTA AÑOS DEL SERVICIO DE ESTUDIOS DEL BANCO DE ESPAÑA
}

\author{
JUAN VELARDE FUERTES \\ Real Academia de Ciencias Morales y Políticas
}

Gracias a un libro reciente de Pablo Martín Aceña ${ }^{1}$ hemos pasado a comprender plenamente la complejidad que tiene el Servicio de Estudios del Banco de España. Por una parte, es nada menos que una institución inmersa en el seno de nuestro Banco Central, en grandísima parte puesta al servicio de la política monetaria española. Sin el Servicio, ésta hoy sería, por fuerza, bastante diferente. De ahí se deriva una influencia muy importante y, por ello, poderosa, en el conjunto de la política española toda. Por otra, es un lugar de investigación de la economía que, desde hace setenta años trabaja, además, con unos niveles muy altos de calidad científica, mídanse éstos como se desee. De ahí se ha acabado desprendiendo que sus funcionarios tengan una especie de nexo común, lo que les convierte, de modo casi automático, en miembros de una escuela. Por supuesto que ésta tiene muchísimo en común con la que se ha denominado Escuela de Madrid ${ }^{2}$, pero, si se quiere, dentro de ésta, pasa a poseer un fuerte espíritu común derivado de una especie de orgullo satisfecho por pertenecer a algo que, en el terreno de la investigación económica de altura, se observa

${ }^{1}$ Pablo Martín Aceña, El Servicio de Estudios del Banco de España, 1930/2000, Banco de España, Madrid, 2000, $10+327$ pp.

2 Me ocupé de la Escuela de Madrid en «Los economistas de la Escuela de Madrid y la Real Academia de Ciencias Morales y Políticas», discurso que está recogido en el volumen Solemne acto de apertura de Curso de las Reales Academias del Instituto de España bajo la presidencia de Sus Majestades los Reyes de España, Real Academia de Ciencias Morales y Políticas, Madrid, 11 de octubre de 1999, pp. 1-71; también, con el título de «La Real Academia de Ciencias Morales y Políticas y la Escuela de Madrid», se recoge lo esencial de ese texto en Torre de los Lujanes, núm. 40, diciembre 1999, pp. 15-27. 
que supera la calidad de la de buena parte de los colegas y grupos existentes. Además, tal orgullo se acentúa porque el Servicio de Estudios tiene ya tal continuidad que les pone al abrigo de riesgos de liquidación de su labor. En tercer término, esta institución es, desde el punto de vista administrativo $-\mathrm{y}$, por supuesto, desde las perspectivas mercantil y laboralun simple fragmento de esa muy amplia realidad que es el Banco de España. Más de un aspecto del mismo se desdibuja si lo separamos de esta institución mucho más moderna que el Banco. A veces esto se olvida al enjuiciar las tareas, que sólo se entienden históricamente, si comprendemos que el Servicio del que ha sido hasta ahora nuestro Instituto emisor, en principio, ni se creó para altas tareas del Estado, ni para que fuese un semillero de estudiosos, sino para mejorar la eficacia de un Banco central que, además, era entonces una sociedad anónima que procuraba maximizar sus beneficios.

Quizá sea importante, en este sentido, consignar que el Servicio de Estudios aparece cuando el Banco de España se ve obligado a dar un viraje, en buena parte a pesar suyo, para poder actuar, al contrario de lo sucedido hasta entonces, en pro de la economía nacional. En realidad, casi coexiste este Servicio con el inicio de un caminar, impuesto por la Ley Prieto de 1931, que conduciría al Banco de España, inexorablemente, a la estatificación por la Ley de 1962. Tiene, pues, poco, o poquísimo, que ver con la etapa anterior a la que cabría titular, con la expresión de Olariaga, El Banco de España, plaga nacional ${ }^{3}$.

Contemplado en su conjunto, se ha de admitir, y mucho más tras la lectura de la obra del profesor Martín Aceña, que es un Servicio que ha de ser analizado tanto para conocer el capitulo de la Economía en el gran libro de la Historia de la ciencia española, como para comprender acon-

${ }^{3}$ Este título tiene que relacionarse con la pregunta que se atribuye a Gumersindo de Azcárate: «¿Es el Banco de España o España del Banco?» ^. Los artículos de Luis Olariaga sobre esto fueron: «El Banco de España, plaga nacional. I. El Estado español autoriza gratuitamente al Banco para que fabrique dinero y luego se lo pide en préstamo, regalándole millones en concepto de interés», en España, núm. 2, 5 de febrero de 1915, año I, pp. 8-9; «El Banco de España, plaga nacional. II. El Banco es el mejor negocio de su clase que existe en Europa y para asegurar su grandes dividendos se violenta la Ley cuando es necesario», en España, núm. 3, 12 de febrero de 1915, año I, pp. 3-4; «El Banco de España, plaga nacional. III. Las ganancias del Banco son causa permanente de que en tiempo normal sea depreciado el dinero español», en España, núm. 4, 19 de febrero de 1915, año I, p. 3; «El Banco de España, plaga nacional. IV. El Banco no cumple su misión fundamental», en España, núm. 5, 26 de febrero de 1915, año I, pp. 8-9; y, finalmente, «El Banco de España, plaga nacional. Comentarios y críticas de la serie», en España, núm. 7, 12 de marzo de 1915, año I, p. 4. 
tecimientos esenciales de la historia de España. He de subrayar desde esta embocadura, que el profesor Martín Aceña actúa de modo admirable en esa siempre difícil tarea de encaje de la explicación del devenir de la Historia de España con la del no menos apasionante mundo de la descripción de las labores científicas de esta institución.

Una obra de la ambición de ésta de Martín Aceña ha de cumplir tres condiciones. La primera, historiar de manera concisa y clara. En segundo lugar, no incurrir en ningún error científico. La tercera, ser capaz de integrar en un cuerpo único lo que -ciencia, política, tarea burocrática normaltiende a disgregarse si no se tiene maestría al relatar lo que ha sucedido. Conviene decir que, tras someter este libro a ese triple contraste, se puede afirmar que lo supera con grado de excelencia.

Puntualicemos algunos aspectos especialmente interesantes. En primer lugar, hay que dejar bien claro que la relación de economistas y estadísticos que presenta en el Servicio Pablo Martín Aceña, nada, o poquísimo, tiene que ver con la Escuela de Flores de Lemus, que parecía dominar la escena española, no sólo hasta la muerte de este economista, sino mucho más allá, a través del grupo del Instituto de Estudios Políticos. Los nombres del Servicio de Estudios que se van presentando bien puntualizados a los lectores son, fundamentalmente, los de Olegario Fernández Baños, Germán Bernácer, Francisco Jainaga, José Larraz, Mariano Sebastián, Antonio de Miguel Martín, Emilio de Figueroa, Ángel Vegas Pérez, Juan Sardá Dexeus, Ángel Madroñero, Luis Ángel Rojo, José Luis Malo de Molina y José Viñals. De todo este conjunto, las únicas excepciones posibles, en el sentido de las relaciones con la escuela de Flores de Lemus, serían los de Mariano Sebastián, Antonio de Miguel y Emilio Figueroa. Pues bien, aun esto sería de modo muy limitado.

Efectivamente Mariano Sebastián pasó por la ordalía de aquella frase de Flores de Lemus, que casi se convirtió en una expresión fundamental de la escuela: «¿Qué es eso de que ha estudiado Vd. ya el Schmoller? Pues vuelva a estudiárselo otra vez», y así varias veces, hasta casi la extenuación del aspirante al ingreso en la escuela. Mariano Sebastián parece haber sido uno de los que no acataron esto sin protesta ${ }^{4}$.

4 La última vez que me encontré con Mariano Sebastián Herrador fue delante de una librería de la que salíamos los dos. Comenzamos a conversar y recorrimos un buen trecho juntos. Me habló de su formación en Valladolid y en Madrid; de sus relaciones vallisoletanas con Onésimo Redondo --yo le di la noticia, y se rió mucho y confirmó mi hipótesis, de que en las Obras completas de éste, en la llamada Edición cronológica. I. Introducción de Narciso García Sánchez. Dirección General de Información. Publicaciones Españolas, 
Por lo que se refiere a Antonio de Miguel, la impresión que queda es la de una admiración de éste hacia Flores de Lemus, que no era correspondida más que con una actitud despectiva. Basta leer el Prólogo que Flores escribió al libro de De Miguel, Introducción a la metodología estadística. Fundamentos de estadística matemática ${ }^{5}$. Siempre, tras leerlo, he dudado si Antonio de Miguel era hombre de humildad franciscana, o bien si, una vez solicitado, no se atrevió a enfurecer a Flores no publicando su original. Quizá la idea de que así, con el nombre de este economista, que no prodigaba su firma, en la portada del libro, atraería a más lectores, endulzaría lo amargo del juicio.

Finalmente, queda el caso de Emilio de Figueroa, que se autoproclamaba discípulo de Flores, pero que jamás lo he encontrado en ninguna de las relaciones de éstos. Asimismo se declaraba discípulo de Bernácer. Ambas cosas a la vez, parecen inverosímiles. La personalidad del profesor Figueroa, que psicológicamente era desconcertante, explica que sin haber tenido relación de discípulo con Flores de Lemus, no sólo se lo atribuyese, sino, además, que se lo creyese $y$, por ello, que sin mentir, defendiese enfáticamente lo que objetiva, no subjetivamente, era una falsedad ${ }^{6}$.

La mayor extrañeza, en este sentido de la ausencia de Flores de Lemus en relación con el Servicio de Estudios del Banco de España, se debe a la persona de quien fue su primer director. Por supuesto, eliminamos de la relación a Francisco Recasens y Mercadé, que había sido designado antes que nadie, a propuesta del ministro de Hacienda, Julio Wais y que

Madrid, 1954, había varios textos publicados en Libertad que se atribuian a Onésimo y que en realidad eran de Mariano Sebastián, a causa de un estropicio de Narciso García Sánchez, que quedaba confirmado en las enfáticas declaraciones de éste en la p. XIII-; de la evolución, peligrosa, del $Y a$; finalmente, de sus poco gratificantes relaciones con Flores de Lemus, quien, una y otra vez, le disuadía de estudiar a Marshall y le recomendaba - más bien habria que decir que le imponia - que trabajase los dos tomos de la Política Social y Economia Politica de Schmoller, en la edición de Imprenta de Henrich y Comp. Editores, Barcelona, 1905, a los que había llegado a odiar. Quedamos en conversar más ampliamente, pero el profesor Sebastián Herrador falleció al poco tiempo.

s Imprenta de Samarás y Cía., Madrid, 1934. El Prólogo lo fecha Flores de Lemus en Madrid, a 27 de junio de 1924.

- Una de las consecuencias de esta curiosa personalidad, que casi podría denominarse de general de la Rovere, de acuerdo con la genial obra de Vittorio de Sica, es que, siendo Figueroa muy trabajador y estudioso, no dejase el menor rastro significativo en el Servicio de Estudios, como se desprende de la lectura de las pp. 114, 115, 134, 137, 140, 144, $174,175,177,180$ y 208 de esta obra de Pablo Martín Aceña. Recuerdo que un día me dijo Luis Ángel Rojo: «-Le he dejado libertad a Figueroa para que haga algo original, lo que quiera; no he conseguido resultado alguno.» Sobre sus méritos, que los tuvo, véase mi nota «Ha fallecido el profesor Emilio de Figueroa Martínez», en $A B C$, núm. 27.074, 4 de diciembre de 1989 , p. 46. 
fue llevado por delante, de inmediato, por el ventarrón de la inestabilidad política de los últimos meses del reinado de Alfonso XIII, agudizado, como es bien sabido, por las consecuencias en la opinión pública de la caída profunda de la peseta, que era la cuestión batallona por aquel entonces. Tras esta aparición fugaz ${ }^{7}$, el designado será Miguel Vidal i Guardiola, que había sido discípulo en el «Grupo de Barcelona», de Flores de Lemus ${ }^{8}$. Por supuesto que Vidal i Guardiola duró poquísimo ${ }^{9}$, pero el que no quede ni rastro ahí de la escuela de Flores, como una hipótesis a contrastar, está en el origen de su nombramiento. Vidal i Guardiola había sido escogido a solicitud de Juan Ventosa i Calvell, el último ministro de Hacienda de Alfonso XIII, como consecuencia de que Cambó hubiese decidido crear un partido de centro. Ventosa, como lugarteniente fiel de éste ${ }^{10}$, ocupó la cartera de Hacienda, en un momento delicado de la salud de Cambó ${ }^{11}$. A este último político le parecía utilísimo el disponer de servicios de estudios económicos. En CHADE había creado el primero que funcionó en España y de él sabemos algunas cosas gracias a Perpiñá Grau ${ }^{12}$. Nada de particular

${ }^{7}$ A Recasens se le eligió el 13 de febrero de 1931 y presentó la dimisión el 17 de febrero de 1931; Pablo Martín Aceña, pp. 20-21. Recasens fue un alto y prestigioso funcionario del Centro de Contratación de Moneda. En este libro de Martín Aceña vemos su rastro en las pp. 64 y 69.

${ }^{8}$ Lo más amplio que he encontrado - con iconografia fotográfica incluida- sobre la escuela $\longrightarrow$ o si se prefiere, sobre el grupo- de Barcelona de Flores de Lemus, está en A. Bausili, Capitalismo siglo xx. Sus crisis y vicisitudes, Buenos Aires, Sudamericana, 1985. Bausili, que era uno de los «hombres de Cambó», ha de añadirse a los nombres de Vidal i Guardiola, Bartolomé Amengual, José María Pi Suñer, Manuel Raventós y Josep María Tallada i Pauli, que se mencionan en la p. 21 de Pablo Martín Aceña.

- Se aprobó su nombramiento por el Consejo General del Banco de España el 27 de febrero de 1931; el 22 de mayo de 1931 presentó la dimisión.

${ }^{10}$ Véase, por ejemplo, para entender esta relación cordialísima, la conversación que sostienen Ventosa y Cambó, en relación con la famosa polémica Alba-Cambó acerca del impuesto extraordinario sobre los beneficios conseguidos en la I Guerra Mundial y sus consecuencias de todo orden; tal como se describe en una colosal biografía de Jesús Pabón, Cambó. 1987-1916, Barcelona, Alpha, 1952, p. 477: «Al salir del Nuevo Club, Cambó y Ventosa - después de conversar con Dato-, se sentían contentos y deseaban luchar; les parecía que recobraban su libertad... escapando a una tentativa de ser denostados... Su estado de espíritu puede resumirse en las siguientes palabras: - ¿Adónde crees tú —preguntaba Ventosa - que nos llevará nuestra trayectoria política? -iQué se yo! —contestó Cambó-. Lo mismo podemos llegar al Poder, que ir a parar a un foso de Montjuich.»

1 En los mentideros madrileños se decía, al enterarse de que se le había pronosticado cáncer de laringe a Cambó: iPobre cáncer!

12 Sobre este asunto de CHADE y Cambó, mis artículos «Perpiñá Grau y el pensamiento estructuralista español», en Revista de Economía Política, núm. 83, septiembre-octubre 1979, pp. 43-57; «Román Perpiñá Grau, en la Real Academia de Ciencias Morales y Políticas», en Cuadernos de Información Económica, núm. 87, junio 1994, pp. 167-176, y «Homenaje al Excmo. Sr. D. Román Perpiñá Grau», en Anales de la Real Academia de Ciencias Morales 
tiene que, tanto a Ventosa como a Vidal i Guardiola, les pareciese casi obvio que el Banco de España debería contar con un Servicio de Estudios. Pero, al ponerlo en marcha, da la impresión de que el círculo de economistas de Cambó, antiguos discípulos de Flores de Lemus, pasó a estar fluctuando entre dos fidelidades: la que se debía al viejo maestro, quien, además, era muy influyente en Madrid, y la que se tenía con el brillante político catalán Francisco de Asís Cambó i Batlle. El problema surgía porque no era posible compatibilizar tales fidelidades porque ambas personalidades no parecían tenerse la menor simpatía a partir de un viejo y ruidoso incidente provocado durante un Gobierno de concentración de Maura ${ }^{13}$. Da la impresión, además, que pronto, incluso el grupo del Servicio de Estudios del Banco de España, al incorporar a su seno, primero a Olegario Fernández Baños y, después, a Bernácer, de algún modo aglutina a economistas que, sin ningún rebozo se han atrevido a criticar ciertas partes del Dictamen de la Comisión del Patrón Oro, al que se consideraba por sus discípulos - y realmente lo era-, una aportación de tan extraordinaria altura que sólo cabía ante ella una especie de silencioso respeto reverencial, que no era exactamente el que habían guardado Fernández Baños y Bernácer. Fernández Baños en su Estudio de las fluctuaciones del cambio de la peseta ${ }^{14}$ corrige rotundamente el Dictamen en un punto erróneo -e importantede éste: que en el período 1921-1928 no existió correlación entre cambio y nivel relativo de precios. La crítica de Bernácer había sido casi feroz. Había puesto en solfa desde su lenguaje «hermético» o el empleo confuso de los términos correlación y causalidad, al papel de la balanza de pagos, en conexión con la evolución de los precios, como determinante del cambio, aparte de considerar equivocadas cosas como la afirmación del Dictamen de que la circulación de la plata era una función de la población y del nivel de precios, sin olvidar el rebatir las afirmaciones del Dictamen sobre el papel de las cuentas corrientes, los billetes de banco, la velocidad de circulación del dinero, y las relaciones de todo ello con el cambio ${ }^{15}$.

y Políticas, núm. 71, curso académico 1993-1994, 1994, año XIVI, pp. 554-565. Parece deducirse de las afirmaciones de Perpiñá que la corte de Cambó no era precisamente algo armónico y límpido.

${ }^{13}$ El incidente con Maura de Cambó, a causa de Flores de Lemus, en Francesc Cambó, Memòries (1876-1931), t. 1, 3. ${ }^{a}$ ed., 1981, Alpha, pp. 342-345.

14 Santiago de Compostela, 1930, especialmente en la p. 34.

15 Germán Bernácer, «Más sobre el patrón oro: el Dictamen de la Comisión oficial», en Revista Nacional de Economia, núm. 87, t. XXIX, septiembre-octubre 1929, año XIV, pp. 195-225, y núm. 88, t. XXIX, noviembre-diciembre 1929, año XIV, pp. 403-428; también, 
La segunda consideración es que muy justamente destaca esta obra la labor de un gran director del Servicio, en tanto en cuanto le orientó adecuadamente para siempre. Es curioso que este galardón haya de otorgarse a quien, aunque nunca tuvo el título, sí de hecho lo ostentó: Olegario Fernández Baños. Esa etapa le merece a Martín Aceña (p. 36) un juicio muy favorable, que algo más adelante sintetiza con justeza así en el capítulo 2 -Asesorando a las autoridades económicas durante la Gran Depresión-: «La actividad desplegada por el Servicio de Estudios durante los años treinta, hasta que la guerra civil quebrase su normal funcionamiento, fue ingente, y de una calidad difícil de exagerar.» Dos obras fundamentales siempre resplandecerán entre las de esa etapa: Ritmo de la crisis económica española en relación con la mundial (1934), que se debía esencialmente a Olegario Fernández Baños (nota 64 de la p. 51) y las balanzas de pagos de la economía española, elaboradas por primera vez por Francisco Jainaga, quien «pereció criminalmente asesinado en los fusilamientos colectivos de Paracuellos del Jarama» (p. 87). Además, una incorporación resultó fundamental: la de Germán Bernácer. Mucho le había costado a éste situarse en un puesto destacado de la Villa y Corte. Para su ascenso había seguido una línea heterodoxa en grado sumo. Por una parte, los estudios y oposiciones en Escuelas de Comercio, pero sesgados hacia la química. Por otra, una extraña apertura a la economía, desde el marco de una especie de ampliación del henrygeorgismo con un énfasis muy marcado en la lucha contra la percepción de tipos de interés. Añádanse las reticencias de Ortega y Gasset a abrirle las páginas de El Sol, su republicanismo unido a un mundo intelectual diferente del madrileño - muy amigos suyos eran Óscar Esplá, que en esa época compondría el Himno rural a la Repuiblica española, y Gabriel Miró-, y su desenfado científico que, como acabamos de ver, ni a Flores de Lemus respetaba. Sobre Bernácer «recayó la obligación de preparar los informes periódicos sobre la coyuntura española y mundial», que «comenzaron el 3 de febrero». Es importante, finalmente, conocer la postura conjunta de los hombres del Servicio «sobre la filosofía económica que desde su misma constitución (éste) defendió» (p. 77). Es importante observar, como inmediatamente se explicará, que éste estableció su prioridad en «la estabilidad de los precios, la cual situaron como objetivo primordial e indiscutible de la política económica y a ella subordinaron sus recomendaciones en materia de cambios y tipos de interés» (Ibidem).

Juan Velarde Fuertes, Flores de Lemus ante la economia española, Madrid, Instituto de Estudios Políticos, 1961, pp. 169-172. 
El «estropicio» —expresión de Olariaga- que produjo en el aspecto científico la Guerra Civil afectó al Banco de España. En Zona republicana, materialmente se disolvió: «Fernández Baños y Bernácer no se sintieron a gusto en la España republicana. El primero preparó pronto su salida al extranjero, objetivo que alcanzó en noviembre de 1937. Empero, cuando regresó a la España nacional no fue recibido con los brazos abiertos, comenzando lo que para el de Badarán sería un largo calvario, que a la postre sólo concluiría con su muerte. Se le restableció en su Cátedra, pero nunca le permitieron reincorporarse a su antiguo destino en el Banco de España ${ }^{16}$. Por su lado, Bernácer aprovechó la primera oportunidad que se le presentó para desertar de sus tareas y refugiarse en su ciudad natal hasta el fin de la guerra. Después, su estrella le deparó mayor suerte que a su colega logronés. El de Alicante retornó al Banco, si bien... su peso en el seno del Servicio disminuyó considerablemente.» Se matiza así el relato, un tanto dramático, de Henri Savall ${ }^{17}$.

En la España nacional, el puesto clave del Servicio pasa a José Larraz $y$, también allí, aparece la figura de su futuro director durante muchos años, Mariano Sebastián Herrador, un hombre muy mal estudiado, probablemente como consecuencia de sus malas relaciones con el muy influyente Manuel de Torres y con todo el grupo de discípulos de Flores de Lemus. Sebastián, sin embargo, es clave para explicar muchos acontecimientos económicos españoles, como, por ejemplo, los primeros pasos del INI, que, como es sabido, fue concebido, inicialmente, por Rodríguez Salmones y por el propio Mariano Sebastián, como un Banco industrial estatificado, proyecto que se alteró, debido a Suanzes, en el paso de la Ley al Reglamento de este Instituto. Con Larraz y su energía, probablemente las cosas hubieran ido, en el Servicio de Estudios del Banco de España, por otros derroteros, pero su rápida sustitución por Mariano Sebastián, quien, por otro lado, como hemos dicho, no se entendía con los economistas más importantes de la Facultad de Ciencias Económicas, y las penosas condiciones económicas del momento, condujeron a lo que, con acierto, denomina Martín Aceña «un Servicio de Estudios, "sin pulso"» (p. 133).

En 1957 todo cambió con la llegada de Sardá. Yo lo he recogido en un artículo en el que procuré indagar sobre los materiales existentes en

${ }^{16}$ De esa desdichada e irritante realidad me he ocupado en el libro Economistas españoles contemporáneos: primeros maestros, Madrid, Espasa Calpe, 1990, pp. 220-222.

17 Henri Savall, Germán Bernácer. La beterodoxia en la economía, trad. de Rafael Pérez Torreblanca; revisión de H. Savall y R. Bernácer, Alicante, Instituto de Estudios Alicantinos, 1983, pp. 25-26. 
la etapa de Mariano Sebastián y en la que le siguió, hasta convertir al Servicio en pieza fundamental para explicar el planteamiento inicial y el éxito posterior del Plan de Estabilización ${ }^{18}$. Siempre me interesó mucho la figura de Sardá. Aún recuerdo la fuerte impresión que me causó su personalidad, que surgía de un hombre delgado, de pequeña estatura, medio hundido en un sofá del salón de reuniones del Consejo Superior Bancario, de voz apagada y con tintes irónicos desde su fuerte acento catalán, un día de finales de los años cuarenta, o quizá ya en 1950. Mucho me lo había ponderado mi maestro Olariaga, y lo reiteró al presentármelo en aquella ocasión. Poco a poco fui dándome cuenta que Olariaga tenía razón, mientras yo pasaba a estudiar trabajos suyos a partir del Spanish prices in the nineteenth Century ${ }^{19}$. Me entusiasmó porque, al trabajar yo el Dictamen de la Comisión del patrón oro, me encontré que con este trabajo se enlazaba con las series del profesor de la Universidad de Chicago, Earl J. Hamilton, que se remontaban en Valencia, Aragón y Navarra hasta el período que va de 1351 al 1500 y para el conjunto de España, para el que transcurre de 1501 a $1800^{20}$. Por otro lado, a través de Serra d'Or tuve noticias más amplias del grupo de S'Agaró, dentro de la singular economía de guerra de Cataluña, que se había escudriñado más de una vez ${ }^{21}$. Después encontraría más datos en una entrevista concedida por Sardá a Baltasar Porcel, pero mientras leía todo eso que se relacionaba entonces con las raíces de la famosa - y disparatada - autogestión obrera yugoslava de Tito, me veía obligado a tener en cuenta ciertas aportaciones de Sardá en Moneda y Crédito y, muy especialmente, su trabajo Nueva dirección del pensamiento económico alemán ${ }^{22}$. Ahí se palpaba una orientación clara hacia la escuela de Viena, así como su simpatía hacia los economistas de Kyklos y su admiración por la figura de Jacques Rueff. Todo esto es lo que sirve para explicar su participación, desde el Servicio de Estudios del Banco de España, en el Plan de Estabilización de 1959. Tuve un trato aún más

${ }^{18}$ Juan Velarde Fuertes, «Sardá en el Banc d'Espanya», en Revista Económica de Catalunya, núm. 5, mayo-agosto 1987 , pp. 69-78.

${ }_{19}$ Publicado en el Quarterly Joumal of Economics, vol. LXII, noviembre de 1948, preludio de su obra La política monetaria y las fluctuaciones de la Economia española en el siglo XIX, Madrid, CSIC, 1948.

${ }^{20}$ Mis indagaciones para tener claras las ideas sobre estas series las he intentado sintetizar en el artículo «Los índices de precios de España: una primera aproximación», en Estadística Española, núm. 145, vol. 42, enero-junio 2000, pp. $43-57$.

${ }_{21}$ Inicié estos trabajos con el Próleg a la obra de Albert Pérez Baró, Trenta mesos de col-lectivisme a Catalumya (1936-1939), Esplugues de Llobregat, Ariel, 1970, pp. 7-19.

${ }_{22}$ Aparecido en Anales de Economía, núm. 35, vol. DX, 1949, y reimpreso en esta misma Revista en 1967-1968, 2." época, núm. 19-24. 
directo con Sardá en las reuniones de la Comisión Consultiva que reunió durante más de un año Laureano López Rodó en su despacho de Comisario del Plan de Desarrollo, en la que, al sentarse en ella también Fabián Estapé, más de una vez el diálogo saltaba del castellano al catalán ${ }^{23}$.

Juan Sardá fue, desde luego, una de las personas claves del Plan de Estabilización de $1959^{24}$. Al hablar con él en más de una de las sesiones de la Real Academia de Ciencias Morales y Políticas, me di cuenta de cómo, de alguna manera, se consideraba injustamente expropiado de esta primogenitura. Por eso pensé que era necesario poner remedio a tan patente injusticia, aclarar su vida, revisar su obra científica y hacer todo eso, al par, desde el rigor y el afecto. Tuve, en este sentido, la fortuna de dar con Carmen Martínez Vela. Reunía admirablemente esta joven profesora de Economía todas las condiciones para entender la figura de Sardá. No sólo descendía de la alta burguesía catalana y, por ello, hablaba catalán, sino que era amiga de la familia - concretamente de una hija - de Sardá. Además era una buena economista y no le arredraba el trabajo tedioso de ir y venir a archivos, de entrevistar a personas en Madrid y Barcelona, y de conversar horas con un Sardá ya viejecito, recluido en su domicilio, quien, como es lógico, confundía a veces fechas y nombres, pero que, al mismo tiempo, proporcionaba informaciones importantes, ideas perfectas sobre acontecimientos, mientras se apasionaba en torno a las concretas posturas científicas, como si estuviera en sus tiempos juveniles. Así es como, poco a poco, se fue articulando el libro Joan Sardá: economista de la profesora Martínez Vela ${ }^{25}$.

Ahora, a más de todo esto, tenemos ese espléndido panorama de Sardá en el Banco de España que se ofrece en el capítulo 5, bajo el título La era de Sardá: 1957-1965. Creo que lo más importante del mismo es su interpretación del abandono de Sardá de su puesto en el Servicio de Estudios del Banco: él soñaba según Fuentes Quintana y Martín Aceña (p. 172), con que a la operación estabilizadora siguiese una «mayor flexibilidad de

${ }^{23}$ Sobre esta Comisión Consultiva, véase también Fabián Estapé, De tots colors. Memóries, Barcelona, Ediciones 62, 3. ${ }^{2} \mathrm{ed}$., 2000, pp. 207.211; también, Juan Velarde Fuertes, «La nueva política económica española y el Informe del Banco Mundial» en el volumen coordinado por Manuel Varela, El Fondo Monetario Internacional, el Banco Mundial y la economia española, 50 aniversario de Bretton Woods, Sociedad Estatal Madrid 94, Madrid, Ediciones Pirámide, 1994, pp. 323-341, y con el mismo título en Cuadernos de Información Económica, núm. 90 , septiembre 1994, pp. 205-211.

${ }^{24}$ Sobre esto véase Fabián Estapé, De tots colors, pp. 110-115; también, Juan Velarde Fuertes, "Juan Sardá Dexeus. In memoriam», en Anales de la Real Academia de Ciencias Morales y Políticas, núm. 73, 1996, año académico 1995-1996, año XLVII, pp. 666-671.

${ }^{25}$ Carmen Martínez Vela, Juan Sardá: economista, Madrid, AC, 2000. 
la economía, más mercado, menos intervenciones, más integración con Europa y, al final, más democracia». Muchos creyeron que así iba a ser, y combatieron porque así fuera. Sardá acabó por abandonar, tras «fustigar la línea, emprendida desde 1963, que en su opinión significaba "un retorno a los desequilibrios y distorsiones amenazadoras del futuro"» aparte de que «su pretensión de que la norma de 1962 facilitara el tránsito del Banco de España al lugar central que le correspondía en el sistema monetario español, no llegó a buen puerto... Posiblemente fuese este hecho uno de los que más contribuyó a desinflar su interés por las labores de asesoramiento» (ibidem).

Queda bien puntualizado en este libro cuál fue el gran y decisivo papel de su sucesor, Ángel Madroñero: llevar adelante una complicada política de transición. Porque, como indica el profesor Martín Aceña, Sardá ni «organizó el Servicio de Estudios; ni se preocupó demasiado por dotarle de medios humanos y materiales; cedió frente a los que obstacularizaron sus proyectos, sin presentar batalla; no quiso distraer su atención de otros menesteres que él consideró prioritarios: erigir el imponente edificio analítico y estadístico que supusieron el Informe anual y el Boletín Estadístico... Por lo demás, lo que la oficina de estudios y sus economistas le deben a Sardá es el programa de trabajo que formuló con el Plan de Estabilización: la lucha por la estabilidad de precios y del tipo de cambio, la cruzada a favor de la ortodoxia financiera y por la conversión del Banco de España en un auténtico Banco central, ejecutor independiente de la política monetaria» (ibidem). Pero para ello era necesario dar una batalla que precisaba de más huestes. Madroñero comprendió que ni en ciencia ni en política éstos son tiempos de los Röntgen, de los Cajal, o si se quiere, de los Churchill o los Maura. Son los equipos científicos y los grandes partidos políticos los que han tomado el relevo. Por eso los de Madroñero «fueron unos años en los cuales desde la oficina de estudios se relanzaron los contactos con el mundo universitario», concretamente «con la Facultad madrileña de Economía (pues nadie)... se había preocupado por mantener abiertas las líneas entre ambas instituciones, que evolucionaron dándose literalmente la espalda» (p. 177). Ese amplio bloque de importantes economistas — véase su censo en las pp. 179-180 - tuvo, en lo doctrinal, una evolución que expone así Martín Aceña (p. 197): «Keynesianos de formación, el lugar donde trabajaban -el Banco central-y los instrumentos que investigaron y sobre los que querían influir, les convirtió, real o aparentemente, en los primeros monetaristas del país.» 
Por supuesto, quien de verdad crea el Servicio de Estudios actual es Luis Ángel Rojo: «Lo que ocurre entre 1971 y 1988 no es sólo la consolidación de un excelente departamento de estudios, sino que el instituto de la plaza de la Cibeles, heredero de aquel Banco de San Carlos nacido en 1782, iuno de los más antiguos del mundo!, asume finalmente sus responsabilidades como autoridad monetaria. Y así, si se nos preguntase cuál ha podido ser la principal contribución del Servicio de Estudios de Luis Ángel Rojo a la economía e incluso a la sociedad española, no dudaríamos en afirmar que por encima de cualquier logro sobresale su decisiva influencia en la metamorfosis experimentada por el Banco de España» (p. 199). Su llegada fue, como se dice en la misma página, «inesperada». Según me informó Luis Coronel de Palma el 12 de julio del 2000, precisamente al preguntarle por esa cuestión, al haberla leído en el recién publicado de Pablo Martín Aceña, me dijo que el primer candidato había sido Enrique Fuentes Quintana. Que éste no había querido y había dado el nombre de Rojo, y que él, a pesar los méritos de éste y de Pedro Martínez Méndez, había asumido, «muy personalmente», me recalcó, la designación de Rojo: - y sigo felicitándome de haberlo hecho» ${ }^{26}$.

En 1988, en medio de una gran agitación política y de la trepidación provocada por nuestro ingreso en el ámbito comunitario y en 1998, con el ingreso de la peseta en el Sistema Monetario Europeo, Luis Ángel Rojo pasó a ser, primero subgobernador y, después, gobernador del Banco emisor. Siempre que una fuerte personalidad abandona un puesto, la sucesión no es fácil. Yo no sé si eso ocurrió en la serie de herederos que se escalonan desde Constantino Lluch (21 de junio de 1988 a 25 de octubre de 1989), José Pérez (octubre 1989-julio 1992), pero sí que hasta José Luis Malo de Molina (31 de julio de 1992), que cierra la relación, no se ha logrado, de nuevo, que las tensiones de la sucesión desapareciesen. Ahora, fuera ya del Banco de España Luis Ángel Rojo, y con la creación del Sistema Europeo de Bancos Centrales y el Banco Central Europeo, es cuando todos vamos a atender, ya en solitario, a la labor de Malo de Molina, que comienza a ser tan importante que pronto, como hubo la era de Fernández Baños, la de Sardá y la de Rojo, justo será hablar de la suya.

En el fondo, el Servicio de Estudios del Banco de España no deja de ser algo así como una creación del gran sueño de los rectores de la política monetaria española. El problema del cambio de la peseta está detrás

${ }^{26}$ Testigo de esta declaración era José Celma; no muchos meses después falleció Luis Coronel de Palma. 
de Fernández Baños; la reconstrucción, tras Larraz; el intervencionismo, tras Mariano Sebastián; la estabilización, tras Sardá; la apertura, coronada con Europa, tras Rojo; la Unión Monetaria Europea, tras Malo de Molina. Todos han vivido esas cuestiones como sueños. Porque, de algún modo es cierto lo que escribió, en 1871, Lewis Carrol en $A$ través del espejo, la segunda parte de Alicia en el país de las maravillas:

«-Ahora está soñando. ¿Con quién sueña? ¿Lo sabes?

«-Nadie lo sabe.

«-Sueña contigo. Y si dejara de soñar, ¿qué sería de ti?

«-No lo sé.

«-Desaparecerías. Eres una figura de sueño. Si se despertara ese Rey, te apagarías como una vela» ${ }^{27}$.

${ }^{27}$ La traducción es la que aparece en ese libro maravilloso de Jorge Luis Borges, Silvina Ocampo y Adolfo Bioy Casares, Antologia de la Literatura Fantástica, Barcelona, Edhasa, 1983, p. 149. 